\title{
The anticentre old open cluster NGC 1883: radial velocity and metallicity
}

\author{
Sandro Villanova, ${ }^{1 \star}$ Gustavo Baume ${ }^{2 \star}$ and Giovanni Carraro ${ }^{1 \star}$ \\ ${ }^{1}$ Dipartimento di Astronomia, Universtià di Padova, vic. Osservatorio 3, Padova, Italy \\ ${ }^{2}$ Facultad de Ciencias Astronómicas y Geofísicas de la UNLP, IALP-CONICET, Paseo del Bosque s/n, La Plata, Argentina
}

Accepted 2007 May 15. Received 2007 May 15; in original form 2007 April 2

\begin{abstract}
Having already reported on the first photometric study of the intermediate-age open cluster NGC 1883, in this paper we present the first spectroscopic multi-epoch investigation of a sample of evolved stars in the same cluster. The aim is to derive the cluster membership, velocity and metallicity, and to discuss recent claims in the literature that NGC 1883 is as metal-poor as globular clusters in the halo. Besides, as it is one of the few outer Galactic disc intermediate-age open clusters known so far, it is an ideal target to improve our knowledge of the Galactic disc radial abundance gradient, which is a basic ingredient for any chemical evolution model of the Milky Way. The new data we have obtained allow us to make the basic parameters of NGC 1883 more reliable. We find that the cluster has a mean metallicity of $[\mathrm{Fe} / \mathrm{H}]=-0.20 \pm 0.22$, from which we infer an age $\left(650_{-70}^{+70} \mathrm{Myr}\right)$ close to that of Hyades and a Galactocentric distance of $12.3_{-0.2}^{+0.4} \mathrm{kpc}$. The metal abundance, kinematics and position make NGC 1883 a genuine outer disc intermediate-age open cluster. We confirm that in the outer Galactic disc the abundance gradient is shallower than in the solar vicinity.
\end{abstract}

Key words: Galaxy: evolution - open clusters and associations: general - open clusters and associations: individual: NGC 1883 - Galaxy: structure.

\section{INTRODUCTION}

NGC 1883 is a northern open cluster, located in the second Galactic quadrant, toward the anticentre direction $\left(\alpha=05^{\mathrm{h}} 25^{\mathrm{m}} \cdot 9, \delta=\right.$ $\left.+46^{\circ} 29^{\prime}, l=163^{\circ} .08, b=+06^{\circ} .16, \mathrm{~J} 2000.0\right)$. This cluster was studied by Carraro et al. (2003, hereafter C03), who presented the first $B V I$ CCD photometric observations suggesting an age of about $1 \mathrm{Gyr}$ and a distance of $4.8 \mathrm{kpc}$ from the Sun.

This would imply a Galactocentric distance larger than $13 \mathrm{kpc}$, and would make NGC 1883 a key object for our understanding of the properties of the outer Galactic disc. However, both age and distance are uncertain because of the unknown metallicity of the cluster.

At the position of NGC 1883, the mean abundance (Friel 1995) should be significantly lower than the solar abundance. Unfortunately, the exact amount of this underabundance is poorly constrained, also in the light of results by Twarog, Ashman \& AnthonyTwarog (1997), Carraro et al. (2004) and Yong, Carney \& Teixera de Almeida (2005), which suggests that the disc abundance gradient for Galactocentric distances larger than $\sim 10 \mathrm{kpc}$ becomes much shallower than in the solar vicinity.

Using solar metallicity $(Z=0.019), \mathrm{C} 03$ estimated $E(B-V)=$ $0.23,(m-M)_{V}=14.00$ for NGC 1883 , and $E(B-V)=0.35$,

\footnotetext{
^E-mail: sandro.villanova@unipd.it (SV); gbaume@fcaglp.fcaglp.unlp. edu.ar (GB); giovanni.carraro@unipd.it (GC)
}

$(m-M)_{V}=14.50$ adopting a lower metal content $(Z=0.008)$. This seems to provide a better fit to the star distribution in the colourmagnitude diagram (CMD).

A more recent study was performed by Tadross (2005, hereafter T05) using C03 BVI data and JHK Two-Micron All-Sky Survey (2MASS) data.

T05 obtained a photometric metallicity of about $[\mathrm{Fe} / \mathrm{H}] \sim-1.1$ $(Z \sim 0.0015)$, much lower than that estimated by $\mathrm{C} 03$, and closer to globular clusters. The disagreement between the two abundance estimations has to be solved in order to obtain reliable parameters for this cluster. In fact, NGC 1883 is one of the few intermediateage open clusters located in the outer Galactic disc. For this reason, it plays an important role in defining the shape of the radial abundance gradient in the external regions of the Galactic disc, which is one of the fundamental constraints of models of chemical evolution (Cescutti et al. 2007).

For this purpose, in this paper we present the first spectroscopic study of the cluster, aimed at finding the member stars from radial velocity measurements, and estimating a reliable value for the metallicity. In Section 2, we illustrate the observation and reduction strategies. In Sections 3 and 4, we discuss the membership and the abundance determination. Section 5 is devoted to a comparison between this paper and previous papers. In Section 6 we deal with a new determination of cluster reddening, distance and age. In Section 7 we compare the observed CMD with a synthetic one. Sections 8 and 9 are devoted to the determination of structural parameters for the cluster (radial profile, luminosity function and mass function). 
Table 1. Observed stars.

\begin{tabular}{lccccccccc}
\hline ID (U-1350-057...) & RA (J2000.0) & Dec. (J2000.0) & $V$ & $B-V$ & $V-I$ & $V-K$ & $\begin{array}{c}R V_{\mathrm{H}}(\mathrm{A}) \\
\left(\mathrm{km} \mathrm{s}^{-1}\right)\end{array}$ & $\begin{array}{c}R V_{\mathrm{H}}(\mathrm{B}) \\
\left(\mathrm{km} \mathrm{s}^{-1}\right)\end{array}$ & $\begin{array}{c}R V_{\mathrm{H}}(\mathrm{C}) \\
\left(\mathrm{km} \mathrm{s}^{-1}\right)\end{array}$ \\
\hline 79024 & $05: 26: 02.01$ & $+46: 29: 26.9$ & 12.30 & 1.85 & 1.89 & 4.32 & 34.4 & 33.0 & 34.4 \\
75374 & $05: 25: 51.69$ & $+46: 29: 35.4$ & 13.25 & 1.78 & 1.79 & 4.00 & -29.7 & -32.5 & -32.1 \\
76058 & $05: 25: 53.66$ & $+46: 26: 20.5$ & 13.30 & 1.61 & 1.70 & 3.70 & -29.8 & -30.3 & -30.7 \\
81797 & $05: 26: 09.92$ & $+46: 25: 55.4$ & 11.85 & 1.87 & 1.94 & 4.38 & 2.7 & 3.0 & 2.7 \\
85248 & $05: 26: 19.92$ & $+46: 31: 41.4$ & 12.61 & 2.18 & 2.60 & 5.43 & -17.9 & -18.5 & -18.4 \\
\hline
\end{tabular}

Finally, in Section 10 we discuss NGC 1833 in the context of the Galactic abundance gradient.

\section{OBSERVATIONS AND DATA REDUCTION}

Medium-high resolution $(R \approx 20000)$ spectra of five candidate evolved stars in the field of NGC 1883 have been obtained using the REOSC echelle spectrograph on board the 1.82-m telescope of the Asiago Astronomical Observatory. The echelle spectrograph works with a Thomson $1024 \times 1024$ CCD and the wavelength coverage for our observations is approximately $4600-6400 \AA$. Details about this instrument are given on the Asiago Observatory website (http://www.oapd.inaf.it/asiago/).

The exposures were $3 \times 15 \mathrm{~min}$ for all stars. To improve the signalto-noise $(\mathrm{S} / \mathrm{N})$ ratio, the three exposures were added reaching $\mathrm{S} / \mathrm{N}$ values of up to 20. Each star was observed during three epochs, 2004 December (A), 2005 January (B) and 2006 January (C), allowing us to establish the binarity of each target.

Data were reduced with the IRAF package, ${ }^{1}$ including bias subtraction, flat-field correction, frame combination, extraction of spectral orders, wavelength calibration, sky subtraction and spectral normalization. By comparing known sky-line positions along the spectra, we obtained an error in wavelength calibration of less than $0.01 \AA$ $\left(<0.5 \mathrm{~km} \mathrm{~s}^{-1}\right)$.

Details of the observed stars are listed in Table 1, while the positions of these targets on CMDs are plotted in Fig. 1. Column 1 of Table 1 gives the ID number of the star according to the USNO 2.0 catalogue, columns 2 and 3 give the coordinates, columns 4-7 give the $V$ magnitude and $B-V, V-I$ and $V-K$ colours, and columns 8-10 give the measured radial velocity at different epochs expressed in $\mathrm{km} \mathrm{s}^{-1}$.

The photometry for our stars was taken from $\mathrm{C} 03$ ( $B, V, I$ magnitudes) and from 2MASS ( $K$ magnitude).

The radial velocity was measured using the IRAF package FXCOR, which cross-correlates the observed spectrum with a template. As a template, we used a synthetic spectrum which has mean atmospheric parameters close to the cluster red giant branch (RGB) stars $\left[T_{\text {eff }}=4500 \mathrm{~K}, \log (g)=3.0,[\mathrm{Fe} / \mathrm{H}]=0.0, v_{\mathrm{t}}=1.5 \mathrm{~km} \mathrm{~s}^{-1}\right] . \mathrm{Fi}-$ nally, the heliocentric correction was applied. The typical error for our measurements is less than $1 \mathrm{~km} \mathrm{~s}^{-1}$.

\section{MEMBERSHIP AND CLUSTER MEAN RADIAL VELOCITY}

We make use of these radial velocities to establish star membership to NGC 1883. First, we notice that each star has radial velocities

\footnotetext{
${ }^{1}$ IRAF is distributed by the National Optical Astronomy Observatory, which is operated by the Association of Universities for Research in Astronomy, Inc., under cooperative agreement with the National Science Foundation.
}

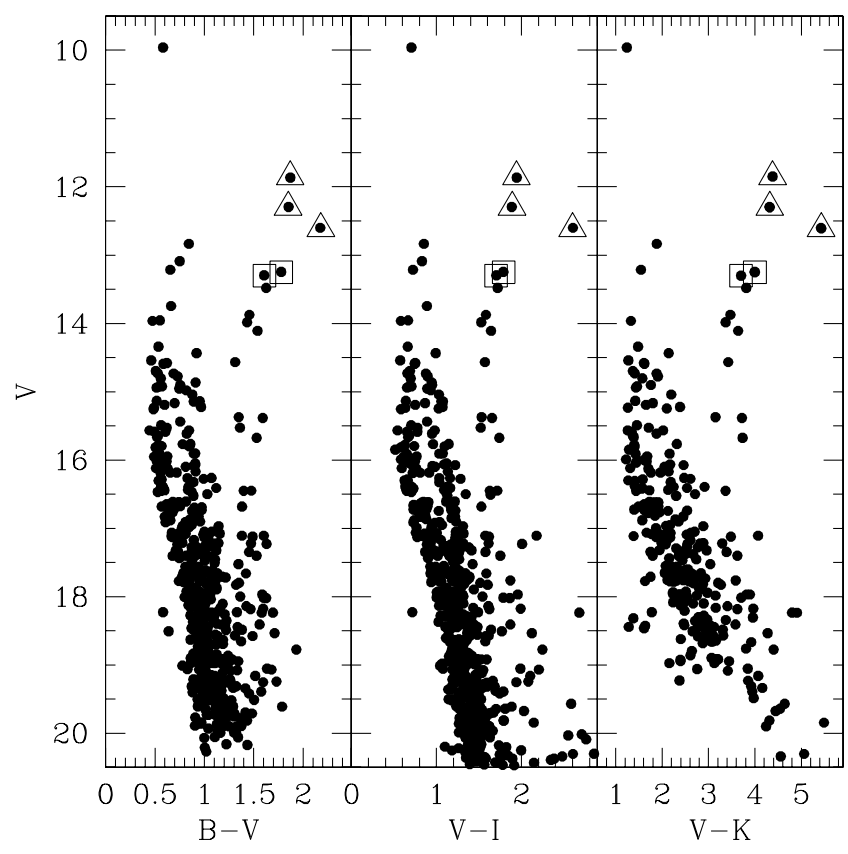

Figure 1. CMD for the stars in the field of NGC 1883. Left: $V$ versus $B-V$ diagram from $\mathrm{C} 03$. Centre: $V$ versus $V-I$ diagram from $C 03$. Right: $V$ versus $V-K$ diagram ( $K$ magnitude from the 2MASS). Open squares are member stars (75374 and 76058), while open triangles are background objects. See text for more details.

constant among the three epochs within the errors. We are led to the conclusion that none of the targets is a short-period binary. The mean radial velocity and membership of all the targets are reported in Table 2, where member stars are indicated by $\mathrm{M}$ and non-members by NM. Only stars 75374 and 76058 have compatible radial velocity, and thus we consider these to be genuine members (open squares in Fig. 1) of the cluster. The average of the velocities of 75374 and 76058 gives us the mean radial velocity of the cluster:

$\left\langle R V_{\text {helio }}\right\rangle=-30.8 \pm 0.6 \mathrm{~km} \mathrm{~s}^{-1}$.

The members stars are used in the following section to obtain the metal content of NGC 1883.

\section{ABUNDANCE MEASUREMENTS}

The $[\mathrm{Fe} / \mathrm{H}]$ content was obtained from the summed spectra of member stars (75374 and 76058) in an interactive way. The most important parameter for abundance determination is $T_{\text {eff }}$, which for our stars can be obtained from dereddened $B-V, V-I$ and $V-K$ colours. However, the reddenings $E(B-V), E(V-I)$ and $E(V-K)$ for the cluster can be obtained only by isochrone fitting, with isochrones having the correct metallicity. For this reason, we first assumed a solar 
Table 2. Mean radial velocity, membership and parameters for the observed stars.

\begin{tabular}{lcccccc}
\hline ID & $\left\langle R V_{\mathrm{H}}\right\rangle\left(\mathrm{km} \mathrm{s}^{-1}\right)$ & Membership & $T_{\mathrm{eff}}(\mathrm{K})$ & $\log (g)$ & $v_{\mathrm{t}}\left(\mathrm{km} \mathrm{s}^{-1}\right)$ & {$[\mathrm{Fe} / \mathrm{H}]$} \\
\hline 79024 & 33.9 & NM & - & - & - & - \\
75374 & -31.4 & M & 4172 & 2.75 & 1.3 & $-0.25 \pm 0.26$ \\
76058 & -30.3 & M & 4462 & 2.75 & 1.3 & $-0.07 \pm 0.46$ \\
81797 & 2.8 & NM & - & - & - & - \\
85248 & -18.3 & NM & - & - & - & - \\
\hline
\end{tabular}

metallicity for our stars, obtaining a first guess for reddening by isochrone fitting. Then, using the $T_{\text {eff }}$ value calculated for this reddening, we measured the metallicity for our stars (see below). Using this metallicity, we obtained a new reddening and a new abundance. This procedure was iterated until convergence was achieved.

$T_{\text {eff }}$ was derived from the colour-[Fe/ $\left.\mathrm{H}\right]$-temperature relation from Alonso, Arribas \& Martínez-Roger (1999) using dereddened colours. The gravity was derived from the value given by the isochrones at the given position of the stars in the CMD, while the microturbulent velocity comes from the relation (Houdashelt, Bell \& Sweigart 2000):

$v_{\mathrm{t}}=2.22-0.322 \times \log (g)$.

The adopted atmospheric parameters for our stars are reported in Table 2 , and the typical random errors are $100 \mathrm{~K}$ for $T_{\text {eff }}, 0.2$ for $\log (g)$ and $0.2 \mathrm{~km} \mathrm{~s}^{-1}$ for $v_{\mathrm{t}}$

We derived metallicity from the equivalent widths of selected spectral lines. The equivalent width was derived by Gaussian fitting of these spectral features. Repeated measurements show a typical error of about $10 \mathrm{~m} \AA$. Because of the low $\mathrm{S} / \mathrm{N}$ and the resolution of our spectra, we could measure only the strongest Fe lines $(\sim 10$ for each star) in the region $\lambda>5500$ to avoid crowding. For this reason, we give only the Fe content of our stars (see Table 2). The local thermodynamic equilibrium (LTE) abundance program MOOG (freely distributed by C. Sneden, University of Texas, Austin, USA; see http://verdi.as.utexas.edu/) was used to determine the metal abundances. Model atmospheres were interpolated from the grid of Kurucz (1992) models by using the values of $T_{\text {eff }}$ and $\log (g)$ of Table 2. The error on the measurements is high (0.2-0.4 dex), but a weighted mean of the obtained metallicities gives a good estimation of the iron content:

$[\mathrm{Fe} / \mathrm{H}]=-0.20 \pm 0.22$.

This value is important for the discussion in the following sections and for comparison with previous works. The errors for iron content that we report here and in Table 2 represent only internal random errors, mainly because of the uncertainty in the equivalent-width measurements. Systematic errors are more difficult to estimate, and are mainly a result of uncertainties in colour $-T_{\text {eff }}$ relations and in reddening. The typical systematic error on the $T_{\text {eff }}$ scale for this temperature range is $100 \mathrm{~K}$, giving a systematic uncertainty on metallicity of 0.02 dex.

\section{COMPARISON WITH PREVIOUS WORK}

First, we compare our results with $\mathrm{C} 03$. C03 estimate a range in metallicity between $Z \sim 0.019([\mathrm{Fe} / \mathrm{H}]=0.0)$ and $Z \sim 0.008$ $([\mathrm{Fe} / \mathrm{H}]=-0.41)$ by isochrone fitting of the turn-off $(\mathrm{TO})$. Our measurements lie exactly in the middle, confirming these results. However, our value largely disagrees with $\mathrm{T} 05$, who gives $[\mathrm{Fe} / \mathrm{H}] \sim$ -1.1 , based on the slope of the RGB stars (Vallenari, Carraro \&
Richichi 2000, and references therein). The method can give a reasonable estimate of the metallicity of a cluster (Tiede, Martini \& Frogel 1997), but $[\mathrm{Fe} / \mathrm{H}] \sim-1.1$ is definitely ruled out by our measurements. The results of T05 can be explained by a poor treatment of stellar contamination. As a matter of fact, background stars always contaminate the field of a cluster, and an identification of the members is needed before applying the RGB-slope method. This is the case for NGC 1883, where a fraction of stars that apparently populate the RGB are background objects, as Fig. 1 unambiguously shows. This contamination changes the real slope of the RGB and the $[\mathrm{Fe} / \mathrm{H}]$ estimate by $\mathrm{T} 05$ is clearly affected by this problem. As shown in Fig. 1 the RGB slope in T05 is mainly based on stars 79024,81797 and 85248 (open triangles), which, according to our radial velocities, are not members. This leads us to the conclusion that the metallicity of T05 is untenable.

\section{CLUSTER FUNDAMENTAL PARAMETERS}

Having an estimate of the metal content $([\mathrm{Fe} / \mathrm{H}]=-0.20)$, we now provide a more reliable estimate of the cluster parameters. We make use of the comparison between the distribution of the stars in the CMDs presented in $\mathrm{C} 03$ and a set of theoretical isochrones from the Padova group (Girardi et al. 2000). In Fig. 2 we superimpose an isochrone with $[\mathrm{Fe} / \mathrm{H}]=-0.20(Z=0.012)$ and an age of 650 Myr. The TO fit is very good both in the $V$ versus $B-V, V$ versus $V-I$ and $V$ versus $V-K$ diagrams, and the shape of the main sequence (MS) is well reproduced. Also, the location of the stars 75374 and 76058 in the RGB is well matched. Using this fitting, we obtained $(m-M)_{V}=14.30$ and $E(B-V)=0.43, E(V-I)=0.52$ and $E(V-K)=1.15$

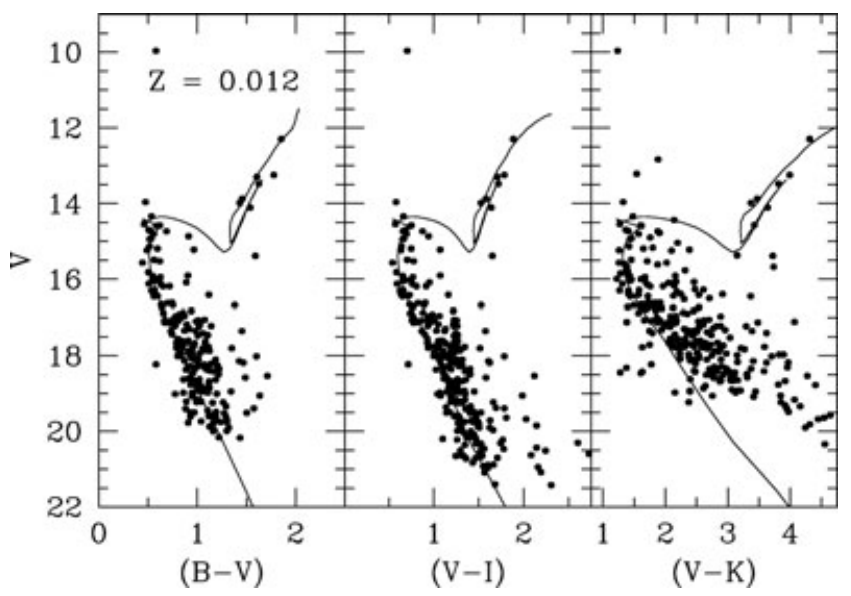

Figure 2. Isochrone fitting of the photometric data using the metallicity obtained in Section 4. Left: $V$ versus $B-V$ diagram from C03. Centre: $V$ versus $V-I$ diagram from $\mathrm{C} 03$. Right: $V$ versus $V-K$ diagram ( $K$ magnitude from the 2MASS). See text for more details. 
The reddening value we obtained is compatible with the maps of Schlegel, Finkbeiner \& Davis (1998), which, in the direction of NGC 1883 , predict a $E(B-V)$ value of 0.55 , all the way to infinity.

NGC 1883 turns out to be located $3.9 \mathrm{kpc}$ from the Sun towards the anticentre direction. This yields from the heliocentric rectangular Galactic coordinates $X=-3.71 \mathrm{kpc}, Y=1.13 \mathrm{kpc}$ and $Z=$ $0.41 \mathrm{kpc}$, and a distance from the Galactic centre of $12.3 \mathrm{kpc}$.

The age we have found in this study is smaller (about $350 \mathrm{Myr}$ ) than in C03, but NGC 1883 can still be considered as one of the few intermediate-age open clusters located in the outer Galactic disc.

The distance estimate is also confirmed by the $V$ magnitude of the red-clump stars (Girardi \& Salaris 2001). They are identified with the group of three stars having $\langle V\rangle=14.0$ and $\langle(B-V)\rangle=1.45$ (see Fig. 2). A $V$ magnitude of 14.0 for red-clump stars gives a distance of $3.6 \pm 0.4 \mathrm{kpc}$ from the Sun, which agrees well with the value we obtained with isochrone fitting.

We interpret the two member stars at $V=13.3$ as bright RGB stars. In fact, the interpretation of these two stars as clump stars would imply a magnitude difference between clump and TO of $\Delta V=2.1$, and, in turn, an age larger than 3 Gyr (Carraro \& Chiosi 1994). An isochrone for this combination of age and metallicity would have too a blue clump, and would produce a serious mismatch of the evolved star distribution. Besides, it would imply a reddening close to zero $[E(B-V) \sim 0.06]$, too small for a cluster, which in this case would lie at $1.7 \mathrm{kpc}$ from the Sun.

\section{SYNTHETIC CMDS}

To better establish the basic parameters of the cluster, we calculated synthetic $V$ versus $B-V$ CMDs. We started from the Girardi et al. (2000) theoretical isochrone of metallicity $Z=0.012$ used for our previous fitting. The isochrone was shifted in apparent magnitude and colour of the amount found in Section 6. The sequence of steps required to simulate a CMD aimed at reproducing the NGC 1883 data are as follows.

(i) The 650-Myr-old isochrone of $Z=0.012$ is used to simulate a cluster with three red-clump stars (see Section 6). Assuming a Kroupa (2001) initial mass function (IMF), in order to reach this number we need an initial cluster mass of about $8.0 \times 10^{2} \mathrm{M}_{\odot}$, which is assumed hereafter. According to the same IMF, we would expect 9-11 stars located beyond the subgiant branch region all the way to the asymptotic giant branch (AGB) region. The exact number and distribution of these stars depend on the simulation seed. Anyway, this range of numbers matches the observations. In fact (see Fig. 2), despite the fact that some contamination is always expected, we count $7 \pm 3$ evolved stars, taking into account Poisson uncertainties.

We have simulated detached binaries, assuming that 30 per cent of the observed objects are binaries with a mass ratio between 0.7 and 1.0. This is in agreement with several estimates for galactic open clusters (Phelps et al. 1994) and with the observational data for NGC 1818 and 1866 in the Large Magellanic Cloud (LMC; Elson et al. 1998; Barmina, Girardi \& Chiosi 2002). The result of such a simulation is shown in Fig. 3(a), where we can see that most of the stars - the single stars - are distributed along the very thin sequence defined by the theoretical isochrone. Binaries appear as both (i) a sequence of objects roughly parallel to the main sequence of single stars and (ii) some more scattered objects in the evolved part of the CMD.

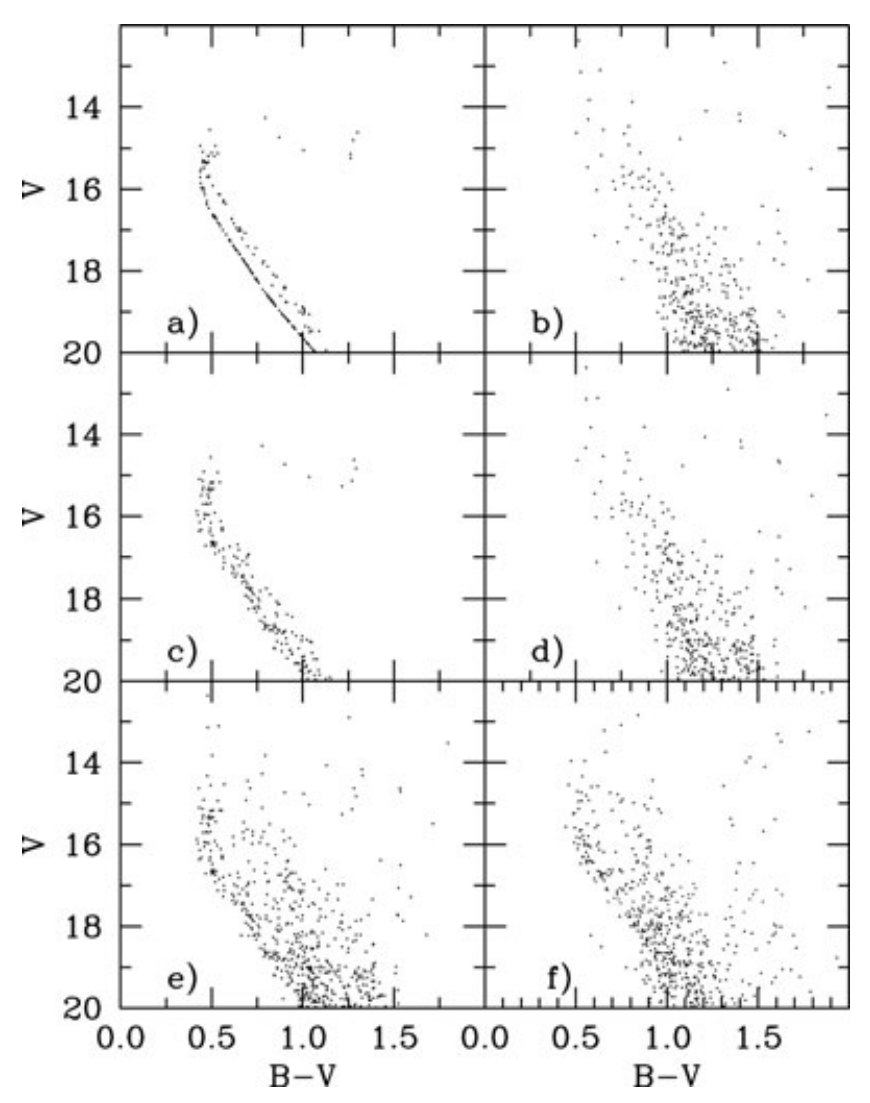

Figure 3. Synthetic CMD diagram for NGC 1883. (a), (b) Simulated CMDs for the cluster and the field. (c), (d) Simulated CMDs for the cluster and the field with photometric errors. (e) Final simulated CMD (cluster + field). (f) The observed CMD.

(ii) In order to estimate the location of foreground and background stars, we use the TRILEGAL ${ }^{2}$ Galaxy model code (Girardi et al. 2005). This includes the several Galactic components - thin and thick discs, halo and an extinction layer - adopting geometric parameters as calibrated by Groenewegen \& Salaris (2001) (for other applications, see Carraro, Subramaniam \& Janes 2006; Baume et al. 2007). The most relevant component in this case is the thin disc, which is modelled by exponential density distributions in both vertical and radial directions. The radial scaleheight is kept fixed $(2.8 \mathrm{kpc})$, whereas the vertical scaleheight $h_{z}$ increases with the stellar age $t$ as

$h_{z}=z_{0}\left(1+t / t_{0}\right)^{\alpha}$

with $z_{0}=95 \mathrm{pc}, t_{0}=4.4 \mathrm{Gyr}$ and $\alpha=1.66$. The simulated field has the same area $\left(8.1 \times 8.1 \operatorname{arcmin}^{2}\right)$ and galactic coordinates ( $\ell=163.08, b=+6.16)$ as that observed for NGC 1883. The results are shown in Fig. 3(b). It is worth noting that, in this direction, most of the Galactic field stars appear in a sort of diagonal sequence in the CMD.

(iii) Within TRILEGAL, there are various options to take reddening into account (Girardi et al. 2005). In the particular case of the simulation we are presenting here, we adopt an exponential dependence of reddening with distance, calibrated at infinity with Schlegel et al. (1998) maps, which provide the reddening along a line of sight all the way to infinity.

\footnotetext{
${ }^{2}$ See http://trilegal.ster.kuleuven.be/cgi-bin/trilegal.
} 
(iv) We then simulate the photometric errors as a function of $V$ magnitude, with typical values derived from our observations. The results are shown separately for cluster and field stars in Figs 3(c) and (d).

(v) The sum of field and cluster simulations is shown in Fig. 3(e). This can be compared directly to the observed data shown in Fig. 3(f).

The comparison of Figs 3(e) and (f) indicates that the selected cluster parameters - age, metallicity, mass, distance, reddening and binary fraction - really lead to an excellent description of the observed CMD, when coupled with the simulated Galactic field. The most noteworthy aspects in this comparison are the location and shape of the turn-off and subgiant branch, which are the features most sensitive to the cluster age.

Of course, there are minor discrepancies between the observed and simulated data, as follows. (i) The simulated cluster is better delineated in the CMD than the data. This may be ascribed to a possible underestimate of the photometric errors in our simulations, and to the possible presence of differential reddening across the cluster. (ii) There is a deficit of simulated field stars, which can be seen more clearly for $V<16$ and $(B-V)<1$. This is caused by the simplified way in which the thin disc is included in the Galactic model. It is represented by means of simple exponentially decreasing stellar densities in both radial and vertical directions, and does not include features such as spiral arms, intervening clusters, etc., which are necessary to correctly describe fields at low galactic latitudes. In any case, the foreground/background simulation we present is only meant to give us an idea of the expected location of field stars in the CMD.

Although these shortcomings in our simulations might probably be eliminated with the use of slightly different prescriptions, they do not affect our main results regarding the choice of cluster parameters. Thus, we conclude that $(m-M)_{V}=14.3, E(B-V)=0.43,650 \mathrm{Myr}$ and $Z=0.012([\mathrm{Fe} / \mathrm{H}]=-0.20)$ represent the cluster parameters well. All these values are uncertain to some extent.

(i) From isochrone fitting, we can estimate a maximum error of 10 per cent (70 Myr) in age. It should be kept in mind, however, that the absolute age value we have derived depends on the choice of evolutionary models, and especially on the prescription for the extent of convective cores. For the stellar masses involved $\left(M_{\mathrm{TO}} \sim\right.$ $1.8 \mathrm{M}_{\odot}$ for NGC 1883), our models (Girardi et al. 2000) include a moderate amount of core overshooting.

(ii) Our best-fitting model corresponds to $E(B-V)=0.43$ and $(m-M)_{V}=14.30$, but typical errors for both measurements can reach $10-15$ per cent.

(iii) The initial mass estimate depends heavily on the choice of IMF, which determines the mass fraction locked into low-mass (unobserved) objects. The value of $8.0 \times 10^{2} \mathrm{M}_{\odot}$ was obtained with a Kroupa (2001) IMF, corrected in the lowest mass interval according to Chabrier (2001) - details are given in Groenewegen \& Salaris (2001) - and should just be considered as a first guess. At present ages, supernovae explosions, stellar mass loss and tidal effects (Lamers et al. 2005) would have reduced this mass by about 40 per cent.

\section{SURFACE DENSITY PROFILE}

According to C03 and Dias et al. (2002), NGC 1883 has a radius of 2.5-3.0 arcmin. This is the distance from the cluster centre where star counts remain flat, reaching the level of the Galactic field. However,

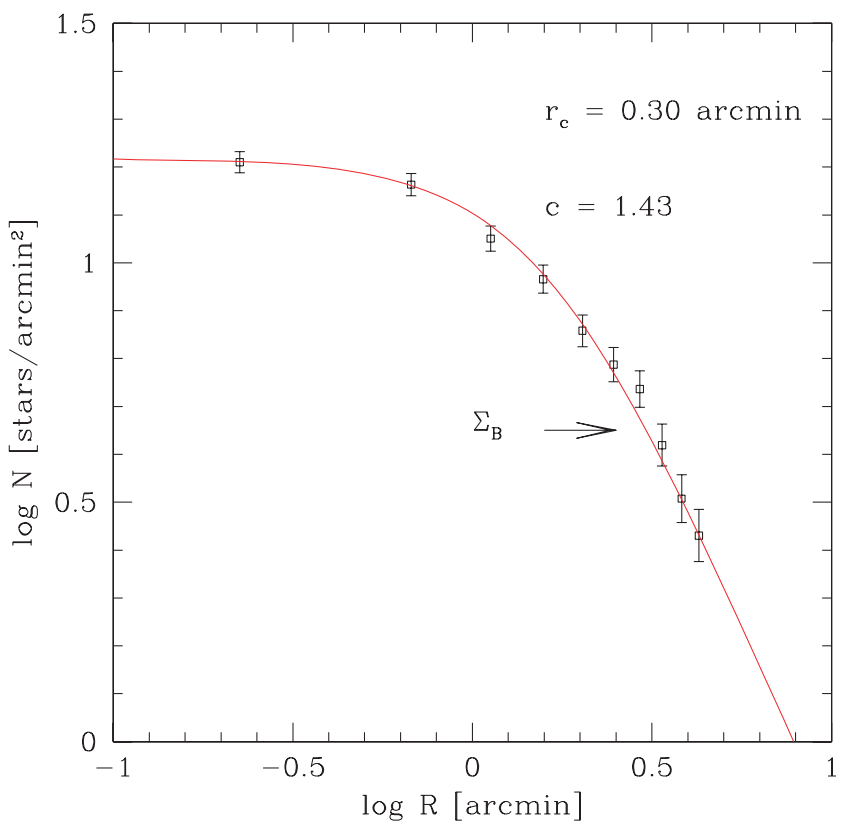

Figure 4. Surface density profile for the cluster. The solid line is a King model for the indicated parameters. $\Sigma_{\mathrm{B}}$ shows the level of the field background.

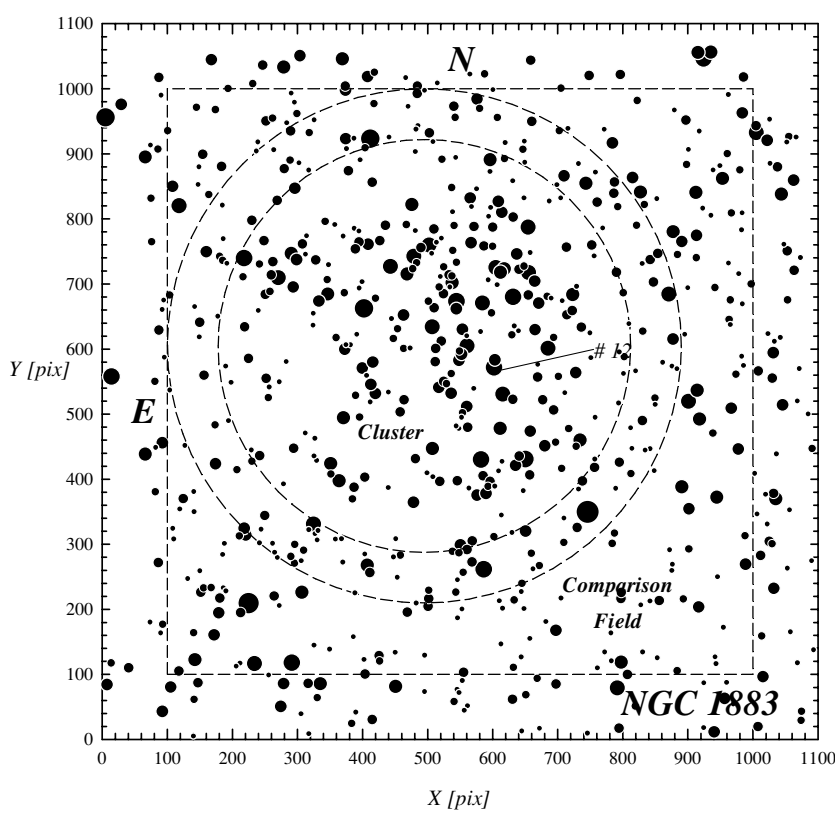

Figure 5. The field used for the cluster luminosity function determination. The cluster region and the comparison field are indicated.

to provide more physical quantities, here we perform a comparison with the King (1962) models (Fig. 4).

With this aim, we first adopt a new cluster centre based on the stellar concentration. $\mathrm{C} 03$ chose star 12 as the centre (see Fig. 5), but a more detailed inspection of the cluster appearance suggests that a more appropriate option for the centre is $X=495, Y=605$ pixels (see Fig. 5), which in turn is $\left(\alpha_{2000}=05^{\mathrm{h}} 25^{\mathrm{m}} 58^{\mathrm{s}} ; \delta_{2000}=\right.$ $\left.+46^{\circ} 29^{\prime} 03^{\prime \prime}\right)$.

By adopting the new cluster centre, we counted the number of stars as a function of the distance from the centre, and corrected these 
counts by background contamination. The choice of the background is illustrated in Fig. 5, and implies that one can expect to encounter $\sim 4.5$ stars $\operatorname{arcmin}^{-2}$ in the direction of NGC 1883 down to $V \sim 20$. This value is in reasonable agreement with the predictions of the TRILEGAL model (Section 7), according to which we expect to find 5.2 stars $\operatorname{arcmin}^{-2}$ towards the direction $l=163.1, b=+6.2$.

Fig. 4 shows star counts together with their Poisson uncertainties. The solid line is the best-fitting King profile, which yields a core radius $r_{\mathrm{c}}=0.30 \operatorname{arcmin}$ and a concentration $c=\log \left(r_{\mathrm{t}} / r_{\mathrm{c}}\right)=1.43$, where $r_{\mathrm{t}}$ is the cluster tidal radius, which works out to be $8.1 \mathrm{arcmin}$, much larger than the region covered by our photometry $(8.1 \times 8.1$ $\operatorname{arcmin}^{2}$ ). These values can be compared with the recent study by Piskunov et al. (2007), who studied the $r_{\mathrm{c}}$ and $r_{\mathrm{t}}$ distributions of a sample of open clusters in the vicinity of the Sun. According to this study, the mean $c$ value of the open cluster is $\sim 0.6$. Therefore, NGC 1883 looks like a poorly concentrated cluster, on the verge of dissolving into the Galactic disc general field.

\section{CLUSTER LUMINOSITY AND MASS FUNCTION}

With the aim of deriving estimates of the integrated luminosity and mass of NGC 1883, we construct the luminosity function (LF) of the cluster.

As a first step, we considered the cluster region and the comparison field: the cluster region is a circle centred in the adopted cluster centre and 2.5 arcmin in radius; the comparison field is a region limited for a circle of 3.11 arcmin in radius and a square of $7.1 \times$ $7.1 \operatorname{arcmin}^{2}$ (see Fig. 5). Both regions were chosen to cover the same area.

We then estimated the completeness of our data as in Baume, Vázquez \& Carraro (2004) and assumed that it is the same across the whole region. This seems to be a good approximation, provided the cluster is not very crowded. The completeness as a function of magnitude $V$ is given in Table 3 .

Moreover, we assume that our comparison field provides a good estimate of the contamination by field interlopers and is valid across all the cluster surface. The results are plotted in Fig. 6.

Here, the completeness, background-corrected stars counts as a function of magnitude are shown together with their Poisson uncertainties. The LF is a rising function over the magnitude range for which our completeness is larger than 50 per cent, except for the last bin, at $V$ larger than 18. This seems to suggest that evaporation of low-mass stars has been important during NGC 1883 evolution, in agreement with the findings in Section 8. As this occurs at the

Table 3. Completeness results in NGC 1883.

\begin{tabular}{cc}
\hline$V$ & Per cent \\
\hline 10.5 & 100.0 \\
11.5 & 100.0 \\
12.5 & 99.9 \\
13.5 & 99.5 \\
14.5 & 97.3 \\
15.5 & 96.1 \\
16.5 & 95.8 \\
17.5 & 90.1 \\
18.5 & 73.4 \\
19.5 & 62.0 \\
20.5 & 41.3 \\
\hline
\end{tabular}

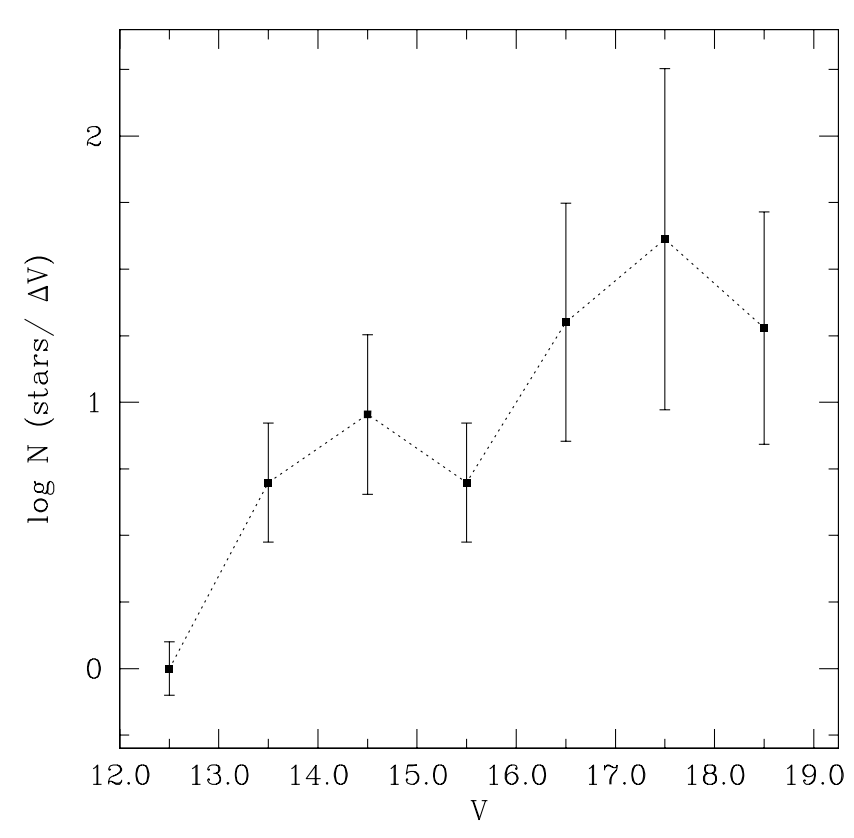

Figure 6. Cluster luminosity function.

limit of our photometry, a deeper study is mandatory to confirm our results.

We also note the presence of a statistically significant depression at $V \sim 15.5$, where the MS in the CMD (see Fig. 1) shows a gap. Such gaps are common in open clusters, and a variety of explanations for their occurrence have been discussed over the years (Rachford \& Canterna 2000).

Apparent magnitudes were then transformed into the absolute magnitudes using the cluster apparent distance modulus $(m-M)_{V}=14.3$. This allows us to compute the integrated absolute magnitude of the cluster by adding up star fluxes. We obtain $M_{V}=-2.4 \pm 0.2$. This value has to be considered as an upper limit, because of the possible presence of several field stars that we are not able to completely remove.

Finally, the $M_{V}$ distribution was converted into a mass function (MF) using the theoretical models of Girardi et al. (2000). The present-day MF is shown in Fig. 7. A linear fit through the points yields $2.1 \pm 0.5$ for the $\alpha$ index, close to the Salpeter 2.35 value. As for the LF, we notice a deficiency of less massive stars, presumably as a result of tidal mass loss. The integration of the present-day MF gives a current total mass for NGC 1883 of about $240 \mathrm{M}_{\odot}$, three times smaller than the mass at birth that we derived in Section 7. This lends further support to a scenario in which substantial mass loss occurred as the cluster orbited the Milky Way.

\section{DISCUSSION AND CONCLUSIONS}

In Fig. 8 we plot the Galactic radial abundance gradient as derived from Friel et al. (2002, hereafter F02) from spectroscopic observations of a sample of open clusters (open squares). We have also added a sample of clusters not observed by F02 (open triangles). We selected these objects from the WEBDA data base ${ }^{3}$ with the condition of having metallicities spectroscopically determined to be homogeneous with F02 and with this paper. We plotted only

\footnotetext{
${ }^{3}$ The WEBDA data base on open clusters can be found at http://www.univie. ac.at/webda/.
} 


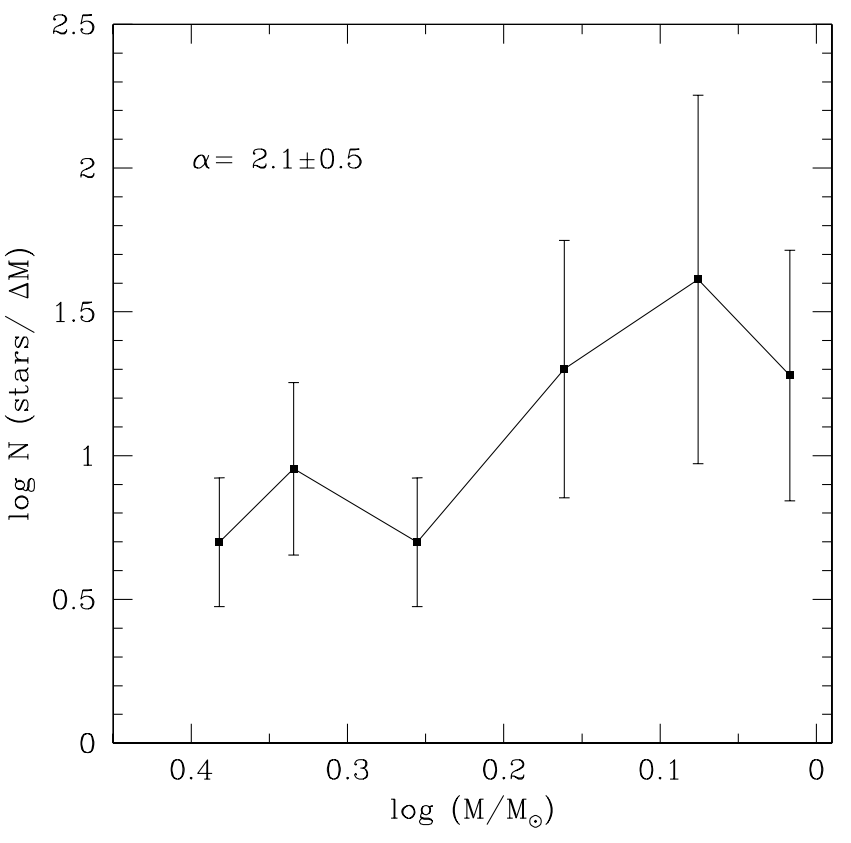

Figure 7. Cluster mass functions.

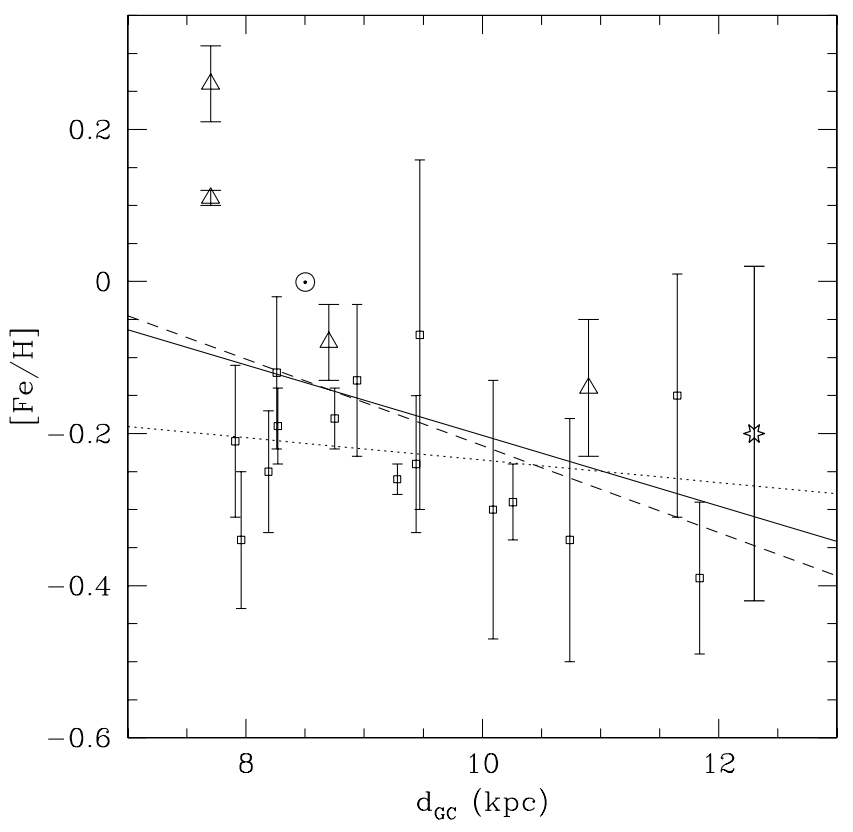

Figure 8. Galactic abundance gradient. Open squares are clusters from Friel et al. (2002), while open triangles are clusters found in the WEBDA data base (see text for more details). The open star is the value for NGC 1883. The position of the Sun $(8.5,0)$ is indicated.

clusters that had an age comparable with NGC $1883(0.5 \leqslant$ Age $\leqslant$ $2.0 \mathrm{Gyr}$, the same range used in F02 to identify the intermediateage cluster sample). The whole sample defines an overall slope of $-0.06 \pm 0.01 \mathrm{dex} \mathrm{kpc}^{-1}$ (dashed line).

The open star represents NGC 1883, which follows the general trend well. In fact, the continuous line, which represents the radial abundance gradient determined by also including NGC 1883, basically coincides with the previous one, having a slope of $-0.05 \pm$

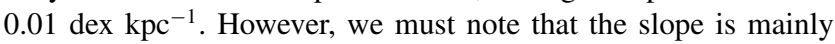
driven by the two innermost metal-rich open clusters (inner tri- angles). If we exclude these points, the gradient is flat, having a value of $-0.01 \pm 0.01 \mathrm{dex} \mathrm{kpc}^{-1}$ (dotted line). Considering also the dispersion of the data and the error of the fit, we are led to the conclusion that the Galactic gradient is flat, at least outside the solar circle. Inside, it probably increases to supersolar metallicity $([\mathrm{Fe} / \mathrm{H}] \sim+0.2)$. However, this last point is not certain, being based only on the metallicities of two clusters, and it needs further investigation.

However, as discussed by F02, the oldest open clusters (Age > 4.0 Gyr) formed in our Galaxy also demonstrate a strong gradient outside the solar circle, as shown in Fig. 3 of F02. This is compatible with the scenario of a primordial pollution of the interstellar matter from which clusters were formed mainly in the inner regions of the Galaxy, following which the chemical enrichment was extended to the outer regions. The result was a present-day homogenization of the chemical content in the Galactic disc, giving a flat gradient as defined by the younger open clusters outside the solar circle. However, this scenario is not valid for the outer regions of the Milky Way.

Carraro et al. (2004) and Yong et al. (2005) found the presence in the outer disc of old open clusters (Age $>4 \mathrm{Gyr}$ ) having a metallicity of about -0.5 dex, well above the value of the gradient as defined by the inner old clusters. This means that probably the outer disc had a peculiar chemical evolution, perhaps driven by the contamination of external objects (dwarf galaxies) cannibalized by the Milky Way.

However, only a few intermediate-age clusters were observed for metallicity determination in the outer disc at medium-high resolution, the majority of these being located close to the Sun.

The addition of NGC 1883 indicates that the gradients remain flat for $0.5 \mathrm{kpc}$ more, suggesting a trend that further studies can possibly confirm.

We finally note that the gradient exhibits a significant scatter. One may wonder whether this solely depends on observational errors, or whether this scatter reflects a true chemical inhomogeneity in the Galactic disc.

Further observations are needed, especially in the not wellexplored Galactic region between 14 and $22 \mathrm{kpc}$ from the Galactic centre. We are actually engaged in such an exploration, with new results being presented in Carraro et al. (2007).

\section{ACKNOWLEDGMENTS}

This paper is based on data collected at the Mount Ekar Observatory, Asiago (Italy). We wish to express our gratitude to the technical staff of the Observatory. In our investigation we made use of WEBDA. Finally, we wish to thank the referee, Bruce Carney, for useful comments.

\section{REFERENCES}

Alonso A., Arribas S., Martínez-Roger C., 1999, A\&AS, 140, 261

Barmina R., Girardi L., Chiosi C., 2002, A\&A, 385, 847

Baume G., Vázquez R. A., Carraro G., 2004, MNRAS, 355, 475

Baume G., Carraro G., Costa E., Méndez R. A., Girardi L., 2007, MNRAS, 375,1077

Carraro G., Chiosi C., 1994, A\&A, 287, 761

Carraro G., Baume G., Villanova S., 2003, MNRAS, 343, 547

Carraro G., Bresolin F., Villanova S., Matteucci F., Patat F., Romaniello M., 2004, AJ, 128, 1676

Carraro G., Subramaniam A., Janes K. A., 2006, MNRAS, 371, 1301

Carraro G., Geisler D., Villanova S., Frinchaboy P. M., Majewsky S. R., 2007, A\&A, submitted

Cescutti G., Matteucci F., François P., Chiappini C., 2007, A\&A, 462, 943 
Chabrier G., 2001, ApJ, 554, 1274

Dias W. S., Alessi B. S., Moitinho A., Lépine J. R. D., 2002, A\&A, 389, 871

Elson R. A. W., Sigurdsson S., Davies M., Hurley J., Gilmore G., 1998, MNRAS, 300, 857

Friel E. D., 1995, ARA\&A, 33, 381

Friel E. D., Janes K. A., Tavarez M., Scott J., Katsanis R., Lotz J., Hong L., Miller N., 2002, AJ, 124, 2693 (F02)

Girardi L., Salaris M., 2001, MNRAS, 323109

Girardi L., Bressan A., Bertelli G., Chiosi C., 2000, A\&AS, 141371

Girardi L., Groenewegen M. A. T., Hatziminaoglou E., da Costa L., 2005, A\&A, 436, 895

Groenewegen M. A. T., Salaris M., 2001, A\&A, 366, 752

Houdashelt M. L., Bell R. A., Sweigart A. V., 2000, AJ, 119, 1448

King I., 1962, AJ, 67, 471

Kroupa P., 2001, MNRAS, 322, 231

Lamers H. J. G. L. M., Gieles M., Bastian N., Baumgardt H., Kharchenko N. V., Portegies Zwart S., 2005, A\&A, 441, 117
Kurucz R. L., 1992, in Barbuy B., Renzini A., eds, Proc. IAU Symp. 149, The Stellar Populations of Galaxies. Kluwer, Dordrecht, p. 225

Phelps R. L., Janes K. A., Montgomery K. A., 1994, AJ, 107, 1079

Piskunov A. E., Schilbach E., Kharchenko N. V., Röser S., Scholz R. D., 2007, A\&A, 468, 151

Rachford B. J., Canterna R., 2000, AJ, 119, 1296

Schlegel D. J., Finkbeiner D. P., Davis M., 1998, ApJ, 500, 525

Tadross A. L., 2005, Bull. Astron. Soc. India, 33, 421 (T05)

Tiede G. P., Martini P., Frogel J. A., 1997, AJ, 114, 694

Twarog B. A., Ashman K. M., Anthony-Twarog B. J., 1997, AJ, 114, 2556

Vallenari A., Carraro G., Richichi A., 2000, A\&A, 353, 147

Yong D., Carney B. W., Teixera de Almeida M. L., 2005, AJ, 130, 597

This paper has been typeset from a $\mathrm{T}_{\mathrm{E}} \mathrm{X} / \mathrm{L} \mathrm{T}_{\mathrm{E}} \mathrm{X}$ file prepared by the author. 